\title{
Orthogonally Additive and Orthogonality Preserving Holomorphic Mappings between $\mathrm{C}^{*}$-Algebras
}

\author{
Jorge J. Garcés, ${ }^{1}$ Antonio M. Peralta, ${ }^{1}$ Daniele Puglisi, ${ }^{2}$ and María Isabel Ramírez ${ }^{3}$ \\ ${ }^{1}$ Departamento de Análisis Matemático, Facultad de Ciencias, Universidad de Granada, 18071 Granada, Spain \\ ${ }^{2}$ Dipartimento di Matematica e Informatica, Università di Catania, 95125 Catania, Italy \\ ${ }^{3}$ Departamento de Algebra y Análisis Matemático, Universidad de Almería, 04120 Almería, Spain
}

Correspondence should be addressed to Antonio M. Peralta; aperalta@ugr.es

Received 1 October 2013; Accepted 27 November 2013

Academic Editor: Ngai-Ching Wong

Copyright (C) 2013 Jorge J. Garcés et al. This is an open access article distributed under the Creative Commons Attribution License, which permits unrestricted use, distribution, and reproduction in any medium, provided the original work is properly cited.

\begin{abstract}
We study holomorphic maps between $C^{*}$-algebras $A$ and $B$, when $f: B_{A}(0, \varrho) \rightarrow B$ is a holomorphic mapping whose Taylor series at zero is uniformly converging in some open unit ball $U=B_{A}(0, \delta)$. If we assume that $f$ is orthogonality preserving and orthogonally additive on $A_{s a} \cap U$ and $f(U)$ contains an invertible element in $B$, then there exist a sequence $\left(h_{n}\right)$ in $B^{* *}$ and Jordan ${ }^{*}$-homomorphisms $\Theta, \widetilde{\Theta}: M(A) \rightarrow B^{* *}$ such that $f(x)=\sum_{n=1}^{\infty} h_{n} \widetilde{\Theta}\left(a^{n}\right)=\sum_{n=1}^{\infty} \Theta\left(a^{n}\right) h_{n}$ uniformly in $a \in U$. When $B$ is abelian, the hypothesis of $B$ being unital and $f(U) \cap \operatorname{inv}(B) \neq \emptyset$ can be relaxed to get the same statement.
\end{abstract}

\section{Introduction}

The description of orthogonally additive $n$-homogeneous polynomial on $C(K)$-spaces and on general $C^{*}$-algebras, developed by Benyamini et al. [1], Pérez-García and Villanueva [2], and Palazuelos et al. [3], respectively (see also $[4,5],[6$, Section 3] and [7]), made functional analysts study and explore orthogonally additive holomorphic functions on $C(K)$-spaces (see $[8,9]$ ) and subsequently on general $C^{*}$ algebras (cf. [10]).

We recall that a mapping $f$ from a $C^{*}$-algebra $A$ into a Banach space $B$ is said to be orthogonally additive on a subset $U \subseteq A$ if for every $a, b$ in $U$ with $a \perp b$, and $a+b \in U$ we have $f(a+b)=f(a)+f(b)$, where elements $a, b$ in $A$ are said to be orthogonal (denoted by $a \perp b$ ) whenever $a b^{*}=b^{*} a=0$. We will say that $f$ is additive on elements having zero product if for every $a, b$ in $A$ with $a b=0$, we have $f(a+b)=f(a)+f(b)$. Having this terminology in mind, the description of all $n$-homogeneous polynomials on a general $C^{*}$-algebra, $A$, which are orthogonally additive on the self-adjoint part, $A_{s a}$, of $A$ reads as follows (see Section 2 for concrete definitions not explained here).
Theorem 1 (see [3]). Let $A$ be a $C^{*}$-algebra and $B$ a Banach space, $n \in \mathbb{N}$, and let $P: A \rightarrow B$ be an $n$-homogeneous polynomial. The following statements are equivalent.

(a) There exists a bounded linear operator $T: A \rightarrow B$ satisfying

$$
P(a)=T\left(a^{n}\right),
$$

for every $a \in A$ and $\|P\| \leq\|T\| \leq 2\|P\|$.

(b) $P$ is additive on elements having zero products.

(c) $P$ is orthogonally additive on $A_{s a}$.

The task of replacing $n$-homogeneous polynomials by polynomials or by holomorphic functions involves a higher difficulty. For example, as noticed by Carando et al. [8, Example 2.2], when $K$ denotes the closed unit disc in $\mathbb{C}$, there is no entire function $\Phi: \mathbb{C} \rightarrow \mathbb{C}$ such that the mapping $h: C(K) \rightarrow C(K), h(f)=\Phi \circ f$ factorizes all degree-2 orthogonally additive scalar polynomials over $C(K)$. Furthermore, similar arguments show that defining $P$ : $C([0,1]) \rightarrow \mathbb{C}, P(f)=f(0)+f(1)^{2}$, we cannot find a triplet 
$\left(\Phi, \alpha_{1}, \alpha_{2}\right)$, where $\Phi: C[0,1] \rightarrow \mathbb{C}$ is a ${ }^{*}$-homomorphism and $\alpha_{1}, \alpha_{2} \in \mathbb{C}$, satisfying that $P(f)=\alpha_{1} \Phi(f)+\alpha_{2} \Phi\left(f^{2}\right)$ for every $f \in C([0,1])$.

To avoid the difficulties commented above, Carando et al. introduce a factorization through an $L_{1}(\mu)$ space. More concretely, for each compact Hausdorff space $K$, a holomorphic mapping of bounded type $f: C(K) \rightarrow \mathbb{C}$ is orthogonally additive if and only if there exist a Borel regular measure $\mu$ on $K$, a sequence $\left(g_{k}\right)_{k} \subseteq L_{1}(\mu)$, and a holomorphic function of bounded type $h: C(K) \rightarrow L_{1}(\mu)$ such that $h(a)=\sum_{k=0}^{\infty} g_{k} a^{k}$ and

$$
f(a)=\int_{K} h(a) d \mu
$$

for every $a \in C(K)$ (cf. [8, Theorem 3.3]).

When $C(K)$ is replaced with a general $C^{*}$-algebra $A$, a holomorphic function of bounded type $f: A \rightarrow \mathbb{C}$ is orthogonally additive on $A_{s a}$ if and only if there exist a positive functional $\varphi$ in $A^{*}$, a sequence $\left(\psi_{n}\right)$ in $L_{1}\left(A^{* *}, \varphi\right)$, and a power series holomorphic function $h$ in $\mathscr{H}_{b}\left(A, A^{*}\right)$ such that

$$
h(a)=\sum_{k=1}^{\infty} \psi_{k} \cdot a^{k}, \quad f(a)=\left\langle 1_{A^{* *}}, h(a)\right\rangle=\int h(a) d \varphi
$$

for every $a$ in $A$, where $1_{A^{* *}}$ denotes the unit element in $A^{* *}$ and $L_{1}\left(A^{* *}, \varphi\right)$ is a noncommutative $L_{1}$-space (cf. [10]).

$\mathrm{A}$ very recent contribution due to $\mathrm{Bu}$ et al. [11] shows that, for holomorphic mappings between $C(K)$ spaces, we can avoid the factorization through an $L_{1}(\mu)$-space by imposing additional hypothesis. Before stating the detailed result, we will set down some definitions.

Let $A$ and $B$ be $C^{*}$-algebras. When $f: U \subseteq A \rightarrow B$ is a map and the condition

$$
\begin{gathered}
a \perp b \Longrightarrow f(a) \perp f(b) \\
(\text { resp., } a b=0 \Longrightarrow f(a) f(b)=0)
\end{gathered}
$$

holds for every $a, b \in U$, we will say that $f$ preserves orthogonality or it is orthogonality preserving (resp., $f$ preserves zero products) on $U$. In the case $A=U$ we will simply say that $f$ is orthogonality preserving (resp., $f$ preserves zero products). Orthogonality preserving bounded linear maps between $\mathrm{C}^{*}$ algebras were completely described in [12, Theorem 17] (see [6] for completeness).

The following Banach-Stone type theorem for zero product preserving or orthogonality preserving holomorphic functions between $C_{0}(L)$ spaces is established by Bu et al. in [11, Theorem 3.4].

Theorem 2 (see [11]). Let $L_{1}$ and $L_{2}$ be locally compact Hausdorff spaces and let $f: B_{C_{0}\left(L_{1}\right)}(0, r) \rightarrow C_{0}\left(L_{2}\right)$ be a bounded orthogonally additive holomorphic function. If $f$ is zero product preserving or orthogonality preserving, then there exist a sequence $\left(\mathcal{O}_{n}\right)$ of open subsets of $L_{2}$, a sequence $\left(h_{n}\right)$ of bounded functions from $L_{2} \cup\{\infty\}$ into $\mathbb{C}$, and a mapping $\varphi: L_{2} \rightarrow L_{1}$ such that for each natural $n$ the function $h_{n}$ is continuous and nonvanishing on $\mathcal{O}_{n}$ and

$$
f(a)(t)=\sum_{n=1}^{\infty} h_{n}(t)(a(\varphi(t)))^{n}, \quad\left(t \in L_{2}\right),
$$

uniformly in $a \in B_{\mathrm{C}_{0}\left(L_{1}\right)}(0, r)$.

The study developed by $\mathrm{Bu}$ et al. is restricted to commutative $\mathrm{C}^{*}$-algebras or to orthogonality preserving and orthogonally additive, $n$-homogeneous polynomials between general $\mathrm{C}^{*}$-algebras. The aim of this paper is to extend their study to holomorphic maps between general $\mathrm{C}^{*}$-algebras. In Section 4, we determine the form of every orthogonality preserving and orthogonally additive holomorphic function from a general $C^{*}$-algebra into a commutative $C^{*}$-algebra (see Theorem 16).

In the wider setting of holomorphic mappings between general $C^{*}$-algebras, we prove the following: let $A$ and $B$ be $C^{*}$-algebras with $B$ unital and let $f: B_{A}(0, \varrho) \rightarrow B$ be a holomorphic mapping whose Taylor series at zero is uniformly converging in some open unit ball $U=B_{A}(0, \delta)$. Suppose $f$ is orthogonality preserving and orthogonally additive on $A_{s a} \cap U$ and $f(U)$ contains an invertible element. Then there exist a sequence $\left(h_{n}\right)$ in $B^{* *}$ and Jordan *homomorphisms $\Theta, \widetilde{\Theta}: M(A) \rightarrow B^{* *}$ such that

$$
f(x)=\sum_{n=1}^{\infty} h_{n} \widetilde{\Theta}\left(a^{n}\right)=\sum_{n=1}^{\infty} \Theta\left(a^{n}\right) h_{n},
$$

uniformly in $a \in U$ (see Theorem 18).

The main tool to establish our main results is a newfangled investigation on orthogonality preserving pairs of operators between $\mathrm{C}^{*}$-algebras developed in Section 3. Among the novelties presented in Section 3, we find an innovating alternative characterization of orthogonality preserving operators between $\mathrm{C}^{*}$-algebras which complements the original one established in [12] (see Proposition 14). Orthogonality preserving pairs of operators are also valid to determine orthogonality preserving operators and orthomorphisms or local operators on $\mathrm{C}^{*}$-algebras in the sense employed by Zaanen [13] and Johnson [14], respectively.

\section{Orthogonally Additive, Orthogonality Preserving, and Holomorphic Mappings on $\mathrm{C}^{*}$-Algebras}

Let $X$ and $Y$ be Banach spaces. Given a natural $n$, a (continuous) $n$-homogeneous polynomial $P$ from $X$ to $Y$ is a mapping $P: X \rightarrow Y$ for which there is a (continuous) $n$-linear symmetric operator $A: X \times \cdots \times X \rightarrow Y$ such that $P(x)=A(x, \ldots, x)$, for every $x \in X$. All polynomials considered in this paper are assumed to be continuous. By a 0 -homogeneous polynomial we mean a constant function. The symbol $\mathscr{P}\left({ }^{n} X, Y\right)$ will denote the Banach space of all continuous $n$-homogeneous polynomials from $X$ to $Y$, with norm given by $\|P\|=\sup _{\|x\| \leq 1}\|P(x)\|$.

Throughout the paper, the word operator will always stand for a bounded linear mapping. 
We recall that, given a domain $U$ in a complex Banach space $X$ (i.e., an open, connected subset), a function $f$ from $U$ to another complex Banach space $Y$ is said to be holomorphic if the Fréchet derivative of $f$ at $z_{0}$ exists for every point $z_{0}$ in $U$. It is known that $f$ is holomorphic in $U$ if and only if for each $z_{0} \in X$ there exists a sequence $\left(P_{k}\left(z_{0}\right)\right)_{k}$ of polynomials from $X$ into $Y$, where each $P_{k}\left(z_{0}\right)$ is $k$-homogeneous, and a neighborhood $V_{z_{0}}$ of $z_{0}$ such that the series,

$$
\sum_{k=0}^{\infty} P_{k}\left(z_{0}\right)\left(y-z_{0}\right)
$$

converges uniformly to $f(y)$ for every $y \in V_{z_{0}}$. Homogeneous polynomials on a $\mathrm{C}^{*}$-algebra $A$ constitute the most basic examples of holomorphic functions on $A$. A holomorphic function $f: X \rightarrow Y$ is said to be of bounded type if it is bounded on all bounded subsets of $X$; in this case its Taylor series at zero, $f=\sum_{k=0}^{\infty} P_{k}$, has infinite radius of uniform convergence, that is, $\lim \sup _{k \rightarrow \infty}\left\|P_{k}\right\|^{1 / k}=0$ (compare [15, Section 6.2], see also [16]).

Suppose $f: B_{X}(0, \delta) \rightarrow Y$ is a holomorphic function and let $f=\sum_{k=0}^{\infty} P_{k}$ be its Taylor series at zero which is assumed to be uniformly convergent in $U=B_{X}(0, \delta)$. Given $\varphi \in Y^{*}$, it follows from Cauchy's integral formula that, for each $a \in U$, we have

$$
\varphi P_{n}(a)=\frac{1}{2 \pi i} \int_{\gamma} \frac{\varphi f(\lambda a)}{\lambda^{n+1}} d \lambda,
$$

where $\gamma$ is the circle forming the boundary of a disc in the complex plane $D_{\mathbb{C}}\left(0, r_{1}\right)$, taken counterclockwise, such that $a+D_{\mathbb{C}}\left(0, r_{1}\right) a \subseteq U$. We refer to [15] for the basic facts and definitions used in this paper.

In this section we will study orthogonally additive, orthogonality preserving, and holomorphic mappings between $\mathrm{C}^{*}$-algebras. We begin with an observation which can be directly derived from Cauchy's integral formula. The statement in the next lemma was originally stated by Carando et al. in [8, Lemma 1.1] (see also [10, Lemma 3]).

Lemma 3. Let $f: B_{A}(0, \varrho) \rightarrow B$ be a holomorphic mapping, where $A$ is a $C^{*}$-algebra and $B$ is a complex Banach space, and let $f=\sum_{k=0}^{\infty} P_{k}$ be its Taylor series at zero, which is uniformly converging in $U=B_{A}(0, \delta)$. Then the mapping $f$ is orthogonally additive on $U$ (resp., orthogonally additive on $A_{s a} \cap U$ or additive on elements having zero product in $U$ ) if and only if all the $P_{k}$ 's satisfy the same property. In such a case, $P_{0}=0$.

We recall that a functional $\varphi$ in the dual of a $C^{*}$-algebra $A$ is symmetric when $\varphi(a) \in \mathbb{R}$, for every $a \in A_{s a}$. Reciprocally, if $\varphi(b) \in \mathbb{R}$ for every symmetric functional $\varphi \in A^{*}$, the element $b$ lies in $A_{s a}$. Having this in mind, our next lemma also is a direct consequence of Cauchy's integral formula and the power series expansion of $f$. A mapping $f: A \rightarrow B$ between $C^{*}$-algebras is called symmetric whenever $f\left(A_{s a}\right) \subseteq$ $B_{s a}$, or equivalently, $f(a)=f(a)^{*}$, whenever $a \in A_{s a}$.

Lemma 4. Let $f: B_{A}(0, \varrho) \rightarrow B$ be a holomorphic mapping, where $A$ and $B$ are $C^{*}$-algebras, and let $f=\sum_{k=0}^{\infty} P_{k}$ be its
Taylor series at zero, which is uniformly converging in $U=$ $B_{A}(0, \delta)$. Then the mapping $f$ is symmetric on $U$ (i.e., $f\left(A_{s a} \cap\right.$ $U) \subseteq B_{s a}$ ) if and only if $P_{k}$ is symmetric (i.e., $P_{k}\left(A_{s a}\right) \subseteq B_{s a}$ ) for every $k \in \mathbb{N} \cup\{0\}$.

Definition 5. Let $S, T: A \rightarrow B$ be a couple of mappings between two $C^{*}$-algebras. One will say that the pair $(S, T)$ is orthogonality preserving on a subset $U \subseteq A$ if $S(a) \perp T(b)$ whenever $a \perp b$ in $U$. When $a b=0$ in $U$ implies $S(a) T(b)=0$ in $B$, we will say that $(S, T)$ preserves zero products on $U$.

We observe that a mapping $T: A \rightarrow B$ is orthogonality preserving in the usual sense if and only if the pair $(T, T)$ is orthogonality preserving. We also notice that $(S, T)$ is orthogonality preserving (on $A_{s a}$ ) if and only if $(T, S)$ is orthogonality preserving (on $A_{s a}$ ).

Our next result assures that the $n$-homogeneous polynomials appearing in the Taylor series of an orthogonality preserving holomorphic mapping between $\mathrm{C}^{*}$-algebras are pairwise orthogonality preserving.

Proposition 6. Let $f: B_{A}(0, \varrho) \rightarrow B$ be a holomorphic mapping, where $A$ and $B$ are $C^{*}$-algebras, and let $f=\sum_{k=0}^{\infty} P_{k}$ be its Taylor series at zero, which is uniformly converging in $U=B_{A}(0, \delta)$. The following statements hold.

(a) The mapping $f$ is orthogonally preserving on $U$ (resp., orthogonally preserving on $A_{s a} \cap U$ ) if and only if $P_{0}=0$ and the pair $\left(P_{n}, P_{m}\right)$ is orthogonality preserving (resp., orthogonally preserving on $A_{\text {sa }}$ ) for every $n, m \in \mathbb{N}$.

(b) The mapping $f$ preserves zero products on $U$ if and only if $P_{0}=0$ and for every $n, m \in \mathbb{N}$, the pair $\left(P_{n}, P_{m}\right)$ preserves zero products.

Proof. (a) The "if" implication is clear. To prove the "only if" implication, let us fix $a, b \in U$ with $a \perp b$. Let us find two positive scalars $r, C$ such that $a, b \in B(0, r)$ and $\|f(x)\| \leq$ $C$ for every $x \in B(0, r) \subset \bar{B}(0, r) \subseteq U$. From the Cauchy estimates we have $\left\|P_{m}\right\| \leq C / r^{m}$, for every $m \in \mathbb{N} \cup\{0\}$. By hypothesis $f(t a) \perp f(t b)$, for every $r>t>0$, hence

$$
\begin{gathered}
P_{0}(t a) P_{0}(t b)^{*}+P_{0}(t a)\left(\sum_{k=1}^{\infty} P_{k}(t b)\right)^{*} \\
+\left(\sum_{k=1}^{\infty} P_{k}(t a)\right)\left(\sum_{k=0}^{\infty} P_{k}(t b)\right)^{*}=0,
\end{gathered}
$$

and by homogeneity

$$
\begin{aligned}
P_{0}(a) P_{0}(b)^{*}= & -P_{0}(a)\left(\sum_{k=1}^{\infty} t^{k} P_{k}(b)\right)^{*} \\
& +\left(\sum_{k=1}^{\infty} t^{k} P_{k}(a)\right)\left(\sum_{k=0}^{\infty} t^{k} P_{k}(b)\right)^{*} .
\end{aligned}
$$

Letting $t \rightarrow 0$, we have $P_{0}(a) P_{0}(b)^{*}=0$. In particular, $P_{0}=0$. 
We will prove by induction on $n$ that the pair $\left(P_{j}, P_{k}\right)$ is orthogonality preserving on $U$ for every $1 \leq j, k \leq n$. Since $f(t a) f(t b)^{*}=0$, we also deduce that

$$
\begin{gathered}
P_{1}(t a) P_{1}(t b)^{*}+P_{1}(t a)\left(\sum_{k=2}^{\infty} P_{k}(t b)\right)^{*} \\
+\left(\sum_{k=2}^{\infty} P_{k}(t a)\right)\left(\sum_{k=1}^{\infty} P_{k}(t b)\right)^{*}=0
\end{gathered}
$$

for every $(\min \{\|a\|,\|b\|\}) / r>t>0$, which implies that

$$
\begin{aligned}
t^{2} P_{1}(a) P_{1}(b)^{*}= & t P_{1}(a)\left(\sum_{k=2}^{\infty} t^{k} P_{k}(b)\right)^{*} \\
& -\left(\sum_{k=2}^{\infty} t^{k} P_{k}(a)\right)\left(\sum_{k=1}^{\infty} t^{k} P_{k}(b)\right)^{*},
\end{aligned}
$$

for every $(\min \{\|a\|,\|b\|\}) / r>t>0$, and hence

$$
\begin{aligned}
\left\|P_{1}(a) P_{1}(b)^{*}\right\| \leq & t C\left\|P_{1}(a)\right\| \sum_{k=2}^{\infty} \frac{\|b\|^{k}}{r^{k}} t^{k-2} \\
& +t C^{2}\left(\sum_{k=2}^{\infty} \frac{\|a\|^{k}}{r^{k}} t^{k-2}\right)\left(\sum_{k=1}^{\infty} \frac{\|b\|^{k}}{r^{k}} t^{k-1}\right) .
\end{aligned}
$$

Taking limit in $t \rightarrow 0$, we get $P_{1}(a) P_{1}(b)^{*}=0$. Let us assume that $\left(P_{j}, P_{k}\right)$ is orthogonality preserving on $U$ for every $1 \leq$ $j, k \leq n$. Following the argument above we deduce that

$$
\begin{aligned}
P_{1}(a) P_{n+1}(b)^{*}+P_{n+1}(a) P_{1}(b)^{*} & -t P_{1}(a)\left(\sum_{j=n+2}^{\infty} t^{j-n-2} P_{j}(b)\right)^{*} \\
& -t \sum_{k=2}^{n} t^{k-2} P_{k}(a)\left(\sum_{j=n+1}^{\infty} t^{j-n-1} P_{j}(b)\right)^{*} \\
& -t P_{n+1}(a)\left(\sum_{j=2}^{\infty} t^{j-2} P_{j}(b)\right)^{*} \\
& -t\left(\sum_{k=n+2}^{\infty} t^{k-n-2} P_{k}(a)\right)\left(\sum_{j=1}^{\infty} t^{j-1} P_{j}(b)\right)^{*},
\end{aligned}
$$

for every $(\min \{\|a\|,\|b\|\}) / r>|t|>0$. Taking limit in $t \rightarrow 0$, we have

$$
P_{1}(a) P_{n+1}(b)^{*}+P_{n+1}(a) P_{1}(b)^{*}=0 \text {. }
$$

Replacing $a$ with $s a(s>0)$ we get

$$
s P_{1}(a) P_{n+1}(b)^{*}+s^{n+1} P_{n+1}(a) P_{1}(b)^{*}=0,
$$

for every $s>0$, which implies that

$$
P_{1}(a) P_{n+1}(b)^{*}=0 \text {. }
$$

In a similar manner we prove that $P_{k}(a) P_{n+1}(b)^{*}=0$, for every $1 \leq k \leq n+1$. The equalities $P_{k}(b)^{*} P_{j}(a)=0(1 \leq$ $j, k \leq n+1)$ follow similarly.

We have shown that for each $n, m \in \mathbb{N}, P_{n}(a) \perp P_{m}(b)$ whenever $a, b \in U$ with $a \perp b$. Finally, taking $a, b \in A$ with $a \perp b$, we can find a positive $\rho$ such that $\rho a, \rho b \in U$ and $\rho a \perp$ $\rho b$, which implies that $P_{n}(\rho a) \perp P_{m}(\rho b)$ for every $n, m \in \mathbb{N}$, witnessing that $\left(P_{n}, P_{m}\right)$ is orthogonality preserving for every $n, m \in \mathbb{N}$.

The proof of (b) follows in a similar manner.

We can obtain now a corollary which is a first step toward the description of orthogonality preserving, orthogonally additive, and holomorphic mappings between $\mathrm{C}^{*}$-algebras.

Corollary 7. Let $f: B_{A}(0, \varrho) \rightarrow B$ be a holomorphic mapping, where $A$ and $B$ are $C^{*}$-algebras and let $f=\sum_{k=0}^{\infty} P_{k}$ be its Taylor series at zero, which is uniformly converging in $U=B_{A}(0, \delta)$. Suppose $f$ is orthogonality preserving and orthogonally additive on (resp., orthogonally additive and zero products preserving) $A_{s a} \cap U$. Then there exists a sequence $\left(T_{n}\right)$ of operators from $A$ into $B$ satisfying that the pair $\left(T_{n}, T_{m}\right)$ is orthogonality preserving on $A_{\text {sa }}$ (resp., zero products preserving on $A_{s a}$ ) for every $n, m \in \mathbb{N}$ and

$$
f(x)=\sum_{n=1}^{\infty} T_{n}\left(x^{n}\right),
$$

uniformly in $x \in U$. In particular every $T_{n}$ is orthogonality preserving (resp., zero products preserving) on $A_{\text {sa }}$. Furthermore, $f$ is symmetric if and only if every $T_{n}$ is symmetric.

Proof. Combining Lemma 3 and Proposition 6, we deduce that $P_{0}=0, P_{n}$ is orthogonally additive on $A_{s a}$, and $\left(P_{n}, P_{m}\right)$ is orthogonality preserving on $A_{s a}$ for every $n, m$ in $\mathbb{N}$. By Theorem 1, for each natural $n$ there exists an operator $T_{n}$ : $A \rightarrow B$ such that $\left\|P_{n}\right\| \leq\left\|T_{n}\right\| \leq 2\left\|P_{n}\right\|$ and

$$
P_{n}(a)=T_{n}\left(a^{n}\right)
$$

for every $a \in A$.

Consider now two positive elements $a, b \in A$ with $a \perp b$ and fix $n, m \in \mathbb{N}$. In this case there exist positive elements $c, d$ in $A$ with $c^{n}=a$ and $d^{m}=b$ and $c \perp d$. Since the pair $\left(P_{n}, P_{m}\right)$ is orthogonality preserving on $A_{s a}$, we have $T_{n}(a)=T_{n}\left(c^{n}\right)=$ $P_{n}(c) \perp P_{m}(d)=T_{m}\left(d^{m}\right)=T_{m}(b)$. Now, noticing that given $a, b$ in $A_{s a}$ with $a \perp b$, we can write $a=a^{+}-b^{-}$and $b=b^{+}-$ $b^{-}$, where $a^{\sigma}$ and $b^{\tau}$ are positive, $a^{+} \perp a^{-}, b^{+} \perp b^{-}$, and $a^{\sigma} \perp$ $b^{\tau}$; for every $\sigma, \tau \in\{+,-\}$, we deduce that $T_{n}(a) \perp T_{m}(b)$. This shows that the pair $\left(T_{n}, T_{m}\right)$ is orthogonality preserving on $A_{\text {sa }}$.

When $f$ is orthogonally additive on $A_{s a}$ and zero products preserving, then the pair $\left(T_{n}, T_{m}\right)$ is zero products preserving on $A_{s a}$ for every $n, m \in \mathbb{N}$. The final statement is clear from Lemma 4.

It should be remarked here that if a mapping $f$ : $B_{A}(0, \delta) \rightarrow B$ is given by an expression of the form in (18) which uniformly converges in $U=B_{A}(0, \delta)$, where $\left(T_{n}\right)$ is a sequence of operators from $A$ into $B$ such that 
the pair $\left(T_{n}, T_{m}\right)$ is orthogonality preserving on $A_{s a}$ (resp., zero products preserving on $A_{s a}$ ) for every $n, m \in \mathbb{N}$, then $f$ is orthogonally additive and orthogonality preserving on $A_{s a} \cap U$ (resp., orthogonally additive on $A_{s a} \cap U$ and zero products preserving).

\section{Orthogonality Preserving Pairs of Operators}

Let $A$ and $B$ be two $C^{*}$-algebras. In this section we will study those pairs of operators $S, T: A \rightarrow B$ satisfying that $S, T$ and the pair $(S, T)$ preserve orthogonality on $A_{s a}$. Our description generalizes some of the results obtained by Wolff in [17] because a (symmetric) mapping $T: A \rightarrow B$ is orthogonality preserving on $A_{s a}$ if and only if the pair $(T, T)$ enjoys the same property. In particular, for every ${ }^{*}$-homomorphism $\Phi$ : $A \rightarrow B$, the pair $(\Phi, \Phi)$ preserves orthogonality. The same statement is true whenever $\Phi$ is a ${ }^{*}$-antihomomorphism, or a Jordan ${ }^{*}$-homomorphism, or a triple homomorphism for the triple product $\{a, b, c\}=(1 / 2)\left(a b^{*} c+c b^{*} a\right)$.

We observe that $S, T$ being symmetric implies that $(S, T)$ is orthogonality preserving on $A_{s a}$ if and only if $(S, T)$ is zero products preserving on $A_{s a}$. We shall present here a newfangled and simplified proof which is also valid for pairs of operators.

Let $a$ be an element in a von Neumann algebra $M$. We recall that the left and right support projections of $a$ (denoted by $l(a)$ and $d(a)$ ) are defined as follows: $l(a)$ (resp., $d(a)$ ) is the smallest projection $p \in M$ (resp., $q \in M$ ) with the property that $p a=a$ (resp., $a q=a$ ). It is known that when $a$ is Hermitian $d(a)=l(a)$ is called the support or range projection of $a$ and is denoted by $s(a)$. It is also known that, for each $a=a^{*}$, the sequence $\left(a^{1 / 3^{n}}\right)$ converges in the strong ${ }^{*}$ topology of $M$ to $s(a)$ (cf. [18, Sections 1.10 and 1.11]).

An element $e$ in a $C^{*}$-algebra $A$ is said to be a partial isometry whenever $e e^{*} e=e$ (equivalently, $e e^{*}$ or $e^{*} e$ is a projection in $A$ ). For each partial isometry $e$, the projections $e e^{*}$ and $e^{*} e$ are called the left and right support projections associated with $e$, respectively. Every partial isometry $e$ in $A$ defines a Jordan product and an involution on $A_{e}(e):=$ $e e^{*} A e^{*} e$ given by $a_{e} b=(1 / 2)\left(a e^{*} b+b e^{*} a\right)$ and $a^{\sharp} e=e a^{*} e$ $\left(a, b \in A_{2}(e)\right)$. It is known that $\left(A_{2}(e), \bullet_{e}, \sharp_{e}\right)$ is a unital $\mathrm{JB}^{*}$-algebra with respect to its natural norm and $e$ is the unit element for the Jordan product $\bullet$.

Every element $a$ in a $C^{*}$-algebra $A$ admits a polar decomposition in $A^{* *}$; that is, $a$ decomposes uniquely as follows: $a=u|a|$, where $|a|=\left(a^{*} a\right)^{1 / 2}$ and $u$ is a partial isometry in $A^{* *}$ such that $u^{*} u=s(|a|)$ and $u u^{*}=s\left(\left|a^{*}\right|\right)(\mathrm{cf}$. [18, Theorem 1.12.1]). Observe that $u u^{*} a=a u^{*} u=u$. The unique partial isometry $u$ appearing in the polar decomposition of $a$ is called the range partial isometry of $a$ and is denoted by $r(a)$. Let us observe that taking $c=r(a)|a|^{1 / 3}$, we have $c c^{*} c=a$. It is also easy to check that for each $b \in A$ with $b=r(a) r(a)^{*} b$ (resp., $\left.b=b r(a)^{*} r(a)\right)$ the condition $a^{*} b=0$ (resp., $\left.b a^{*}=0\right)$ implies $b=0$. Furthermore, $a \perp b$ in $A$ if and only if $r(a) \perp r(b)$ in $A^{* *}$.

We begin with a basic argument in the study of orthogonality preserving operators between $C^{*}$-algebras whose proof is inserted here for completeness reasons. Let us recall that for every $C^{*}$-algebra $A$, the multiplier algebra of $A, M(A)$, is the set of all elements $x \in A^{* *}$ such that for each $A x, x A \subseteq A$. We notice that $M(A)$ is a $C^{*}$-algebra and contains the unit element of $A^{* *}$.

Lemma 8. Let $A$ and $B$ be $C^{*}$-algebras and let $S, T: A \rightarrow B$ be a pair of operators.

(a) The pair $(S, T)$ preserves orthogonality (on $A_{s a}$ ) if and only if the pair $\left(\left.S^{* *}\right|_{M(A)},\left.T^{* *}\right|_{M(A)}\right)$ preserves orthogonality (on $M(A)_{s a}$ ).

(b) The pair $(S, T)$ preserves zero products (on $A_{s a}$ ) if and only if the pair $\left(\left.S^{* *}\right|_{M(A)},\left.T^{* *}\right|_{M(A)}\right)$ preserves zero products (on $M(A)_{s a}$ ).

Proof. (a) The "if" implication is clear. Let $a, b$ be two elements in $M(A)$ with $a \perp b$. We can find two elements $c$ and $d$ in $M(A)$ satisfying $c c^{*} c=a, d d^{*} d=b$, and $c \perp d$. Since $c x c \perp d y d$, for every $x, y$ in $A$, we have $T(c x c) \perp T(d y d)$ for every $x, y \in A$. By Goldstine's theorem we find two bounded nets $\left(x_{\lambda}\right)$ and $\left(y_{\mu}\right)$ in $A$, converging in the weak ${ }^{*}$ topology of $A^{* *}$ to $c^{*}$ and $d^{*}$, respectively. Since $T\left(c x_{\lambda} c\right) T\left(d y_{\mu} d\right)^{*}=T\left(d y_{\mu} d\right)^{*} T\left(c x_{\lambda} c\right)=0$, for every $\lambda, \mu$, $T^{* *}$ is weak*-continuous, the product of $A^{* *}$ is separately weak ${ }^{*}$-continuous, and the involution of $A^{* *}$ is also weak ${ }^{*}$ continuous, we get $T^{* *}\left(c c^{*} c\right) T^{* *}\left(d d^{*} d\right)=T^{* *}(a) T^{* *}(b)^{*}=$ $0=T^{* *}(b)^{*} T^{* *}(a)$ and hence $T^{* *}(a) \perp T^{* *}(b)$, as desired.

The proof of (b) follows by a similar argument.

Proposition 9. Let $S, T: A \rightarrow B$ be operators between $C^{*}$ algebras such that $(S, T)$ is orthogonality preserving on $A_{s a}$. Let us denote $h:=S^{* *}(1)$ and $k:=T^{* *}(1)$. Then the identities,

$$
\begin{gathered}
S(a) T\left(a^{*}\right)^{*}=S\left(a^{2}\right) k^{*}=h T\left(\left(a^{2}\right)^{*}\right)^{*}, \\
T\left(a^{*}\right)^{*} S(a)=k^{*} S\left(a^{2}\right)=T\left(\left(a^{2}\right)^{*}\right)^{*} h, \\
S(a) k^{*}=h T\left(a^{*}\right)^{*}, \quad k^{*} S(a)=T\left(a^{*}\right)^{*} h,
\end{gathered}
$$

hold for every $a \in A$.

Proof. By Lemma 8, we may assume, without loss of generality, that $A$ is unital. (a) for each $\varphi \in B^{*}$, the continuous bilinear form $V_{\varphi}: A \times A \rightarrow \mathbb{C}, V_{\varphi}(a, b)=\varphi\left(S(a) T\left(b^{*}\right)^{*}\right)$ is orthogonal; that is, $V_{\varphi}(a, b)=0$, whenever $a b=0$ in $A_{s a}$. By Goldstein's theorem [19, Theorem 1.10], there exist functionals $\omega_{1}, \omega_{2} \in A^{*}$ satisfying that

$$
V_{\varphi}(a, b)=\omega_{1}(a b)+\omega_{2}(b a),
$$

for all $a, b \in A$. Taking $b=1$ and $a=b$ we have

$$
\begin{aligned}
& \varphi\left(S(a) k^{*}\right)=V_{\varphi}(a, 1)=V_{\varphi}(1, a)=\varphi\left(h T(a)^{*}\right), \\
& \varphi\left(S(a) T(a)^{*}\right)=\varphi\left(S\left(a^{2}\right) k^{*}\right)=\varphi\left(h T\left(a^{2}\right)^{*}\right),
\end{aligned}
$$

for every $a \in A_{s a}$, respectively. Since $\varphi$ was arbitrarily chosen, we get, by linearity, $S(a) k^{*}=h T\left(a^{*}\right)^{*}$ and $S(a) T\left(a^{*}\right)^{*}=$ $S\left(a^{2}\right) k^{*}=h T\left(\left(a^{2}\right)^{*}\right)^{*}$, for every $a \in A$. The other identities follow in a similar way but replacing $V_{\varphi}(a, b)=\varphi\left(S(a) T\left(b^{*}\right)^{*}\right)$ with $V_{\varphi}(a, b)=\varphi\left(T\left(b^{*}\right)^{*} S(a)\right)$. 
Lemma 10. Let $J_{1}, J_{2}: A \rightarrow B$ be Jordan *-homomorphism between $C^{*}$-algebras. The following statements are equivalent.

(a) The pair $\left(J_{1}, J_{2}\right)$ is orthogonality preserving on $A_{s a}$.

(b) The identity

$$
\begin{aligned}
& J_{1}(a) J_{2}(a)=J_{1}\left(a^{2}\right) J_{2}^{* *}(1)=J_{1}^{* *}(1) J_{2}\left(a^{2}\right), \\
& \text { holds for every } a \in A_{s a} \text {, } \\
& \text { (c) The identity, }
\end{aligned}
$$

$$
J_{1}^{* *}(1) J_{2}(a)=J_{1}(a) J_{2}^{* *}(1)
$$

holds for every $a \in A_{s a}$.

Furthermore, when $J_{1}^{* *}$ is unital, $J_{2}(a)=J_{1}(a) J_{2}^{* *}(1)=$ $J_{2}^{* *}(1) J_{1}(a)$, for every $a$ in $A$.

Proof. The implications (a) $\Rightarrow$ (b) $\Rightarrow$ (c) have been established in Proposition 9. To see (c) $\Rightarrow($ a), we observe that $J_{i}(x)=J_{i}^{* *}(1) J_{i}(x) J_{i}^{* *}(1)=J_{i}(x) J_{i}^{* *}(1)=J_{i}^{* *}(1) J_{i}(x)$, for every $x \in A$. Therefore, given $a, b \in A_{s a}$ with $a \perp b$, we have $J_{1}(a) J_{2}(b)=J_{1}(a) J_{1}^{* *}(1) J_{2}(b)=J_{1}(a) J_{1}(b) J_{2}^{* *}(1)=0$.

In [17, Proposition 2.5], Wolff establishes a uniqueness result for ${ }^{*}$-homomorphisms between $\mathrm{C}^{*}$-algebras showing that for each pair $(U, V)$ of unital ${ }^{*}$-homomorphisms from a unital $C^{*}$-algebra $A$ into a unital $C^{*}$-algebra $B$, the condition $(U, V)$ orthogonality preserving on $A_{s a}$ implies $U=V$. This uniqueness result is a direct consequence of our previous lemma.

Orthogonality preserving pairs of operators can be also used to rediscover the notion of orthomorphism in the sense introduced by Zaanen in [13]. We recall that an operator $T$ on a $C^{*}$-algebra $A$ is said to be an orthomorphism or a band preserving operator when the implication $a \perp b \Rightarrow$ $T(a) \perp b$ holds for every $a, b \in A$. We notice that when $A$ is regarded as an $A$-bimodule, an operator $T: A \rightarrow A$ is an orthomorphism if and only if it is a local operator in the sense used by Johnson in [14, Section 3]. Clearly, an operator $T: A \rightarrow A$ is an orthomorphism if and only if $\left(T, I d_{A}\right)$ is orthogonality preserving. The following noncommutative extension of [13, Theorem 5] follows from Proposition 9.

Corollary 11. Let $T$ be an operator on a $C^{*}$-algebra $A$. Then $T$ is an orthomorphism if and only if $T(a)=T^{* *}(1) a=a T^{* *}(1)$, for every $a$ in $A$; that is, $T$ is a multiple of the identity on $A$ by an element in its center.

We recall that two elements $a$, and $b$ in a JB ${ }^{*}$-algebra $A$ are said to operator commute in $A$ if the multiplication operators $M_{a}$ and $M_{b}$ commute, where $M_{a}$ is defined by $M_{a}(x):=a \circ x$. That is, $a$ and $b$ operator commute if and only if $(a \circ x) \circ b=$ $a \circ(x \circ b)$ for all $x$ in $A$. A useful result in Jordan theory assures that self-adjoint elements $a$ and $b$ in $A$ generate a $\mathrm{JB}^{*}$-subalgebra that can be realized as a $\mathrm{JC}^{*}$-subalgebra of some $B(H)$ (compare [20]) and, under this identification, $a$ and $b$ commute as elements in $L(H)$ whenever they operator commute in $A$, equivalently, $a^{2} \circ b=2(a \circ b) \circ a-a^{2} \circ b$ (cf. Proposition 1 in [21]).
The next lemma contains a property which is probably known in $\mathrm{C}^{*}$-algebra, we include an sketch of the proof because we were unable to find an explicit reference.

Lemma 12. Let e be a partial isometry in a $C^{*}$-algebra $A$ and let $a$, and $b$ be two elements in $A_{2}(e)=e e^{*} A e^{*} e$. Then $a, b$ operator commute in the $J B^{*}$-algebra $\left(A_{2}(e), \bullet_{e}, \sharp_{e}\right)$ if and only if $a e^{*}$ and be operator commute in the JB*-algebra $\left(A_{2}\left(e e^{*}\right), \bullet_{e e^{*}}, \#_{e e^{*}}\right)$, where $x \bullet_{e e^{*}} y=x \circ y=(1 / 2)(x y+$ $y x)$, for every $x, y \in A_{2}\left(e e^{*}\right)$. Furthermore, when $a$ and $b$ are hermitian elements in $\left(A_{2}(e), \bullet_{e}, \#_{e}\right), a$, and $b$ operator commute if and only if ae $e^{*}$ and be commute in the usual sense (i.e., $a e^{*} b e^{*}=b e^{*} a e^{*}$ ).

Proof. We observe that the mapping $R_{e^{*}}:\left(A_{2}(e), \bullet_{e}\right) \rightarrow$ $\left(A_{2}\left(e e^{*}\right), \bullet e e^{*}\right), x \mapsto x e^{*}$, is a Jordan ${ }^{*}$-isomorphism between the above JB ${ }^{*}$-algebras. So, the first equivalence is clear. The second one has been commented before.

Our next corollary relies on the following description of orthogonality preserving operators between $\mathrm{C}^{*}$-algebras obtained in [12] (see also [6]).

Theorem 13 (see [12, Theorem 17], [6, Theorem 4.1 and Corollary 4.2]). If $T$ is an operator from a $C^{*}$-algebra $A$ into another $C^{*}$-algebra $B$ the following are equivalent.

(a) $T$ is orthogonality preserving (on $A_{s a}$ ).

(b) There exists a unital Jordan *-homomorphism $J$ : $M(A) \rightarrow B_{2}^{* *}(r(h))$ such that $J(x)$ and $h=T^{* *}(1)$ operator commute and

$$
T(x)=h \bullet_{r(h)} J(x), \quad \text { for every } x \in A,
$$

where $M(A)$ is the multiplier algebra of $A, r(h)$ is the range partial isometry of $h$ in $B^{* *}, B_{2}^{* *}(r(h))=$ $r(h) r(h)^{*} B^{* *} r(h)^{*} r(h)$, and $\bullet_{r(h)}$ is the natural product making $B_{2}^{* *}(r(h))$ a JB* -algebra.

Furthermore, when $T$ is symmetric, $h$ is hermitian and hence $r(h)$ decomposes as orthogonal sum of two projections in $B^{* *}$.

Our next result gives a new perspective for the study of orthogonality preserving (pairs of) operators between $\mathrm{C}^{*}$ algebras.

Proposition 14. Let $A$ and $B$ be $C^{*}$-algebras. Let $S, T: A \rightarrow$ $B$ be operators and let $h=S^{* *}(1)$ and $k=T^{* *}(1)$. Then the following statements hold.

(a) The operator $S$ is orthogonality preserving if and only if there exist two Jordan ${ }^{*}$-homomorphisms $\Phi, \widetilde{\Phi}$ : $M(A) \rightarrow B^{* *}$ satisfying $\Phi(1)=r(h) r(h)^{*}, \widetilde{\Phi}(1)=$ $r(h)^{*} r(h)$, and $S(a)=\Phi(a) h=h \widetilde{\Phi}(a)$, for every $a \in A$.

(b) $S, T$ and $(S, T)$ are orthogonality preserving on $A_{s a}$ if and only if the following statements hold.

(b1) There exist Jordan ${ }^{*}$-homomorphisms $\Phi_{1}, \widetilde{\Phi}_{1}$, $\Phi_{2}, \widetilde{\Phi}_{2}: M(A) \rightarrow B^{* *}$ satisfying $\Phi_{1}(1)=$ $r(h) r(h)^{*}, \widetilde{\Phi}_{1}(1)=r(h)^{*} r(h), \Phi_{2}(1)=r(k)$ 
$r(k)^{*}, \widetilde{\Phi}_{2}(1)=r(k)^{*} r(k), S(a)=\Phi_{1}(a) h=$ $h \widetilde{\Phi}_{1}(a)$, and $T(a)=\Phi_{2}(a) k=k \widetilde{\Phi}_{2}(a)$, for every $a \in A$.

(b2) The pairs $\left(\Phi_{1}, \Phi_{2}\right)$ and $\left(\widetilde{\Phi}_{1}, \widetilde{\Phi}_{2}\right)$ are orthogonality preserving on $A_{s a}$.

Proof. The "if" implications are clear in both statements. We will only prove the "only if" implication.

(a) By Theorem 13, there exists a unital Jordan *homomorphism $J_{1}: M(A) \rightarrow B_{2}^{* *}(r(h))$ such that $J_{1}(x)$ and $h$ operator commute in the $\mathrm{JB}^{*}$-algebra $\left(B_{2}^{* *}(r(h)), \cdot r(h)\right)$ and

$$
S(x)=h \bullet_{r(a)} J_{1}(a) \quad \text { for every } a \in A .
$$

Fix $a \in A_{s a}$. Since $h$ and $J_{1}(a)$ are hermitian elements in $\left(B_{2}^{* *}(r(h)), \bullet_{r(h)}\right)$ which operator commute, Lemma 12 assures that $h r(h)^{*}$ and $J_{1}(a) r(h)^{*}$ commute in the usual sense of $B^{* *}$; that is,

$$
h r(h)^{*} J_{1}(a) r(h)^{*}=J_{1}(a) r(h)^{*} h r(h)^{*}
$$

or equivalently,

$$
h r(h)^{*} J_{1}(a)=J_{1}(a) r(h)^{*} h .
$$

Consequently, we have

$$
S(a)=h \bullet_{r(h)} J_{1}(a)=h r(h)^{*} J_{1}(a)=J_{1}(a) r(h)^{*} h,
$$

for every $a \in A$. The desired statement follows by considering $\Phi_{1}(a)=J_{1}(a) r(h)^{*}$ and $\widetilde{\Phi}_{1}(a)=$ $r(h)^{*} J_{1}(a)$.

(b) The statement in (b1) follows from (a). We will prove (b2). By hypothesis, given $a, b$ in $A_{s a}$ with $a \perp b$, we have

$$
\begin{aligned}
0 & =S(a) T(b)^{*}=\left(h \widetilde{\Phi}_{1}(a)\right)\left(k \widetilde{\Phi}_{2}(b)\right)^{*} \\
& =h \widetilde{\Phi}_{1}(a) \widetilde{\Phi}_{2}(b)^{*} k^{*} .
\end{aligned}
$$

Having in mind that $\widetilde{\Phi}_{1}(A) \subseteq r(h)^{*} r(h) B^{* *}$ and $\widetilde{\Phi}_{2}(A) \subseteq B^{* *} r(k)^{*} r(k)$, we deduce that $\widetilde{\Phi}_{1}(a) \widetilde{\Phi}_{2}(b)^{*}=0$ (compare the comments before Lemma 8), as we desired. In a similar fashion we prove $\widetilde{\Phi}_{2}(b)^{*} \widetilde{\Phi}_{1}(a)=0, \Phi_{2}(b)^{*} \Phi_{1}(a)=0=\Phi_{1}(a) \Phi_{2}(b)^{*}$.

\section{Holomorphic Mappings Valued in a Commutative $\mathrm{C}^{*}$-Algebra}

The particular setting in which a holomorphic function is valued in a commutative $C^{*}$-algebra $B$ provides enough advantages to establish a full description of the orthogonally additive, orthogonality preserving, and holomorphic mappings which are valued in $B$.
Proposition 15. Let $S, T: A \rightarrow B$ be operators between $C^{*}$ algebras with $B$ commutative. Suppose that $S, T$ and $(S, T)$ are orthogonality preserving, and let us denote $h=S^{* *}(1)$ and $k=T^{* *}(1)$. Then there exists a Jordan *-homomorphism $\Phi: M(A) \rightarrow B^{* *}$ satisfying $\Phi(1)=r(|h|+|k|), S(a)=\Phi(a) h$, and $T(a)=\Phi(a) k$, for every $a \in A$.

Proof. Let $\Phi_{1}, \widetilde{\Phi}_{1}, \Phi_{2}, \widetilde{\Phi}_{2}: M(A) \rightarrow B^{* *}$ be the Jordan * homomorphisms satisfying (b1) and (b2) in Proposition 14. By hypothesis, $B$ is commutative, and hence $\Phi_{i}=\widetilde{\Phi}_{i}$ for every $i=1,2$ (compare the proof of Proposition 14). Since the pair $\left(\Phi_{1}, \Phi_{2}\right)$ is orthogonality preserving on $A_{s a}$, Lemma 10 assures that

$$
\Phi_{1}^{* *}(1) \Phi_{2}(a)=\Phi_{1}(a) \Phi_{2}^{* *}(1)
$$

for every $a \in A_{s a}$. In order to simplify notation, let us denote $p=\Phi_{1}^{* *}(1)$ and $q=\Phi_{2}^{* *}(1)$.

We define an operator $\Phi: M(A) \rightarrow B^{* *}$, given by

$$
\Phi(a)=p q \Phi_{1}(a)+p(1-q) \Phi_{1}(a)+q(1-p) \Phi_{2}(a) .
$$

Since $p \Phi_{2}(a)=\Phi_{1}(a) q$, it can be easily checked that $\Phi$ is a Jordan *-homomorphism such that $S(a)=\Phi(a) h$ and $T(a)=\Phi(a) k$, for every $a \in A$.

Theorem 16. Let $f: B_{A}(0, \varrho) \rightarrow B$ be a holomorphic mapping, where $A$ and $B$ are $C^{*}$-algebras with $B$ commutative and let $f=\sum_{k=0}^{\infty} P_{k}$ be its Taylor series at zero, which is uniformly converging in $U=B_{A}(0, \delta)$. Suppose $f$ is orthogonality preserving and orthogonally additive on $A_{s a} \cap$ $U$ (equivalently, orthogonally additive on $A_{s a} \cap U$ and zero products preserving). Then there exist a sequence $\left(h_{n}\right)$ in $B^{* *}$ and a Jordan ${ }^{*}$-homomorphism $\Phi: M(A) \rightarrow B^{* *}$ such that

$$
f(x)=\sum_{n=1}^{\infty} h_{n} \Phi\left(a^{n}\right)=\sum_{n=1}^{\infty} h_{n} \Phi\left(a^{n}\right),
$$

uniformly in $a \in U$.

Proof. By Corollary 7 , there exists a sequence $\left(T_{n}\right)$ of operators from $A$ into $B$ satisfying that the pair $\left(T_{n}, T_{m}\right)$ is orthogonality preserving on $A_{s a}$ (equivalently, zero products preserving on $A_{s a}$ ) for every $n, m \in \mathbb{N}$ and

$$
f(x)=\sum_{n=1}^{\infty} T_{n}\left(x^{n}\right),
$$

uniformly in $x \in U$. Denote $h_{n}=T_{n}^{* *}(1)$

We will prove now the existence of the Jordan * homomorphism $\Phi$. We prove, by induction, that for each natural $n$, there exists a Jordan ${ }^{*}$-homomorphism $\Psi_{n}$ : $M(A) \rightarrow B^{* *}$ such that $r\left(\Psi_{n}(1)\right)=r\left(\left|h_{1}\right|+\cdots+\left|h_{n}\right|\right)$ and $T_{k}(a)=h_{k} \Psi_{n}(a)$ for every $k \leq n, a \in A$. The statement for $n=1$ follows from Corollary 7 and Proposition 14. Let us assume that our statement is true for $n$. Since for every $k, m$ in $\mathbb{N}, T_{k}, T_{m}$, and the pair $\left(T_{k}, T_{m}\right)$ are orthogonality preserving, we can easily check that $T_{n+1}, T_{1}+\cdots+T_{n}$ and $\left(T_{n+1}, T_{1}+\cdots+\right.$ $\left.T_{n}\right)=\left(T_{n+1},\left(h_{1}+\cdots+h_{n}\right) \Psi_{n}\right)$ are orthogonality preserving. 
By Proposition 15, there exists a Jordan *-homomorphism $\Psi_{n+1}: M(A) \rightarrow B^{* *}$ satisfying $r\left(\Psi_{n+1}(1)\right)=r\left(\left|h_{1}\right|+\cdots+\right.$ $\left.\left|h_{n}\right|+\left|h_{n+1}\right|\right), T_{n+1}(a)=h_{n+1} \Psi_{n+1}\left(a^{n+1}\right)$ and $\left(T_{1}+\cdots+T_{n}\right)(a)=$ $\left(h_{1}+\cdots+h_{n}\right) \Psi_{n+1}(a)$ for every $a \in A$. Since, for each $1 \leq k \leq n$,

$$
\begin{aligned}
h_{k} \Psi_{n+1}(a) & =h_{k} r\left(\left|h_{1}\right|+\cdots+\left|h_{n}\right|+\left|h_{n+1}\right|\right) \Psi_{n+1}(a) \\
& =h_{k} r\left(\left|h_{1}\right|+\cdots+\left|h_{n}\right|\right) \Psi_{n+1}(a) \\
& =h_{k} r\left(\left|h_{1}\right|+\cdots+\left|h_{n}\right|\right) \Psi_{n}(a)=h_{k} \Psi_{n}(a)=T_{k}(a),
\end{aligned}
$$

for every $a \in A$, as desired.

Let us consider a free ultrafilter $\mathcal{U}$ on $\mathbb{N}$. By the BanachAlaoglu theorem, any bounded set in $B^{* *}$ is relatively weak ${ }^{*}$ compact, and thus the assignment $a \mapsto \Phi(a):=w^{*}-$ $\lim _{\mathcal{U}} \Psi_{n}(a)$ defines a Jordan ${ }^{*}$-homomorphism from $M(A)$ into $B^{* *}$. If we fix a natural $k$, we know that $T_{k}(a)=h_{k} \Psi_{n}(a)$, for every $n \geq k$ and $a \in A$. Then it can be easily checked that $T_{k}(a)=h_{k} \Phi(a)$, for every $a \in A$, which concludes the proof.

The Banach-Stone type theorem for orthogonally additive, orthogonality preserving, and holomorphic mappings between commutative $\mathrm{C}^{*}$-algebras, established in Theorem 2 (see [11, Theorem 3.4]), is a direct consequence of our previous result.

\section{Banach-Stone Type Theorems for Holomorphic Mappings between General $\mathrm{C}^{*}$-Algebras}

In this section we deal with holomorphic functions between general $\mathrm{C}^{*}$-algebras. In this more general setting we will require additional hypothesis to establish a result in the line of the above Theorem 16.

Given a unital $C^{*}$-algebra $A$, the symbol $\operatorname{inv}(A)$ will denote the set of invertible elements in $A$. The next lemma is a technical tool which is needed later. The proof is left to the reader and follows easily from the fact that $\operatorname{inv}(A)$ is an open subset of $A$.

Lemma 17. Let $f: B_{A}(0, \varrho) \rightarrow B$ be a holomorphic mapping, where $A$ and $B$ are $C^{*}$-algebras with $B$ unital and let $f=\sum_{k=0}^{\infty} P_{k}$ be its Taylor series at zero, which is uniformly converging in $U=B_{A}(0, \delta)$. Let us assume that there exists $a_{0} \in U$ with $f\left(a_{0}\right) \in \operatorname{inv}(B)$. Then there exists $m_{0} \in \mathbb{N}$ such that $\sum_{k=0}^{m_{0}} P_{k}\left(a_{0}\right) \in \operatorname{inv}(B)$.

We can now state a description of those orthogonally additive, orthogonality preserving, and holomorphic mappings between $\mathrm{C}^{*}$-algebras whose image contains an invertible element.

Theorem 18. Let $f: B_{A}(0, \varrho) \rightarrow B$ be a holomorphic mapping, where $A$ and $B$ are $C^{*}$-algebras with $B$ unital and let $f=\sum_{k=0}^{\infty} P_{k}$ be its Taylor series at zero, which is uniformly converging in $U=B_{A}(0, \delta)$. Suppose $f$ is orthogonality preserving and orthogonally additive on $A_{s a} \cap U$ and $f(U) \cap \operatorname{inv}(B) \neq \emptyset$.
Then there exist a sequence $\left(h_{n}\right)$ in $B^{* *}$ and Jordan *homomorphisms $\Theta, \widetilde{\Theta}: M(A) \rightarrow B^{* *}$ such that

$$
f(a)=\sum_{n=1}^{\infty} h_{n} \widetilde{\Theta}\left(a^{n}\right)=\sum_{n=1}^{\infty} \Theta\left(a^{n}\right) h_{n}
$$

uniformly in $a \in U$.

Proof. By Corollary 7 there exists a sequence $\left(T_{n}\right)$ of operators from $A$ into $B$ satisfying that the pair $\left(T_{n}, T_{m}\right)$ is orthogonality preserving on $A_{s a}$ for every $n, m \in \mathbb{N}$ and

$$
f(x)=\sum_{n=1}^{\infty} T_{n}\left(x^{n}\right),
$$

uniformly in $x \in U$.

Now, Proposition 14 (a), applied to $T_{n}(n \in \mathbb{N})$, implies the existence of sequences $\left(\Phi_{n}\right)$ and $\left(\widetilde{\Phi}_{n}\right)$ of Jordan *homomorphisms from $M(A)$ into $B^{* *}$ satisfying $\Phi_{n}(1)=$ $r\left(h_{n}\right) r\left(h_{n}\right)^{*}, \widetilde{\Phi}_{n}(1)=r\left(h_{n}\right)^{*} r\left(h_{n}\right)$, where $h_{n}=T_{n}^{* *}(1)$, and

$$
T_{n}(a)=\Phi_{n}(a) h_{n}=h_{n} \widetilde{\Phi}_{n}(a),
$$

for every $a \in A, n \in \mathbb{N}$. Moreover, from Proposition 14 (b), the pairs $\left(\Phi_{n}, \Phi_{m}\right)$ and $\left(\widetilde{\Phi}_{n}, \widetilde{\Phi}_{m}\right)$ are orthogonality preserving on $A_{s a}$, for every $n, m \in \mathbb{N}$.

Since $f(U) \cap \operatorname{inv}(B) \neq \emptyset$, it follows from Lemma 17 that there exists a natural $m_{0}$ and $a_{0} \in A$ such that

$$
\sum_{k=1}^{m_{0}} P_{k}\left(a_{0}\right)=\sum_{k=1}^{m_{0}} \Phi_{k}\left(a_{0}^{k}\right) h_{k}=\sum_{k=1}^{m_{0}} h_{k} \widetilde{\Phi}_{k}\left(a_{0}^{k}\right) \in \operatorname{inv}(B) .
$$

We claim that $r\left(r\left(h_{1}\right)^{*} r\left(h_{1}\right)+\cdots+r\left(h_{m_{0}}\right)^{*} r\left(h_{m_{0}}\right)\right)=1$ in $B^{* *}$. Otherwise, we find a nonzero projection $q \in B^{* *}$ satisfying

$$
r\left(r\left(h_{1}\right)^{*} r\left(h_{1}\right)+\cdots+r\left(h_{m_{0}}\right)^{*} r\left(h_{m_{0}}\right)\right) q=0 .
$$

Since $r\left(h_{i}\right)^{*} r\left(h_{i}\right) \leq r\left(r\left(h_{1}\right)^{*} r\left(h_{1}\right)+\cdots+r\left(h_{m_{0}}\right)^{*} r\left(h_{m_{0}}\right)\right)$, this would imply that

$$
\left(\sum_{k=1}^{m_{0}} P_{k}\left(a_{0}\right)\right) q=\left(\sum_{k=1}^{m_{0}} \Phi_{k}\left(a_{0}^{k}\right) h_{k}\right) q=0,
$$

contradicting that $\sum_{k=1}^{m_{0}} P_{k}\left(a_{0}\right)=\sum_{k=1}^{m_{0}} \Phi_{k}\left(a_{0}^{k}\right) h_{k}$ is invertible in $B$.

Consider now the mapping $\Psi=\sum_{k=1}^{m_{0}} \widetilde{\Phi}_{k}$. It is clear that, for each natural $n, \Psi, \widetilde{\Phi}_{n}$, and the pair $\left(\Psi, \widetilde{\Phi}_{n}\right)$ are orthogonality preserving. Applying Proposition 14 (b), we deduce the existence of Jordan ${ }^{*}$-homomorphisms $\Theta, \widetilde{\Theta}, \Theta_{n}, \widetilde{\Theta}_{n}$ : $M(A) \rightarrow B^{* *}$ such that $\left(\Theta, \Theta_{n}\right)$ and $\left(\widetilde{\Theta}, \widetilde{\Theta}_{n}\right)$ are orthogonality preserving, $\Theta(1)=r(k) r(k)^{*}, \widetilde{\Theta}(1)=r(k)^{*} r(k)$, $\Theta_{n}(1)=r\left(h_{n}\right) r\left(h_{n}\right)^{*}, \widetilde{\Theta}_{n}(1)=r\left(h_{n}\right)^{*} r\left(h_{n}\right)$,

$$
\begin{gathered}
\Psi(a)=\Theta(a) k=k \widetilde{\Theta}(a), \\
\widetilde{\Phi}_{n}(a)=\Theta_{n}(a) r\left(h_{n}\right)^{*} r\left(h_{n}\right)=r\left(h_{n}\right)^{*} r\left(h_{n}\right) \widetilde{\Theta}_{n}(a),
\end{gathered}
$$


for every $a \in A$, where $k=\Psi(1)=r\left(h_{1}\right)^{*} r\left(h_{1}\right)+$ $\cdots+r\left(h_{m_{0}}\right)^{*} r\left(h_{m_{0}}\right)$. The condition $r(k)=1$, proved in the previous paragraph, shows that $\Theta(1)=1$. Thus, since $\left(\widetilde{\Theta}, \widetilde{\Theta}_{n}\right)$ is orthogonality preserving, the last statement in Lemma 10 proves that

$$
\widetilde{\Theta}_{n}(a)=\widetilde{\Theta}_{n}(1) \widetilde{\Theta}(a)=\widetilde{\Theta}(a) \widetilde{\Theta}_{n}(1),
$$

for every $a \in A, n \in \mathbb{N}$. The above identities guarantee that

$$
\widetilde{\Phi}_{n}(a)=\Theta(a) r\left(h_{n}\right)^{*} r\left(h_{n}\right)=r\left(h_{n}\right)^{*} r\left(h_{n}\right) \widetilde{\Theta}(a),
$$

for every $a \in A, n \in \mathbb{N}$.

A similar argument to the one given above, but replacing $\widetilde{\Phi}_{k}$ with $\Phi_{k}$, shows the existence of a Jordan ${ }^{*}$ homomorphism $\Theta: M(A) \rightarrow B^{* *}$ such that

$$
\Phi_{n}(a)=\Theta(a) r\left(h_{n}\right) r\left(h_{n}\right)^{*}=r\left(h_{n}\right) r\left(h_{n}\right)^{*} \Theta(a),
$$

for every $a \in A, n \in \mathbb{N}$, which concludes the proof.

\section{Conflict of Interests}

The authors declare that there is no conflict of interests regarding the publication of this paper.

\section{Acknowledgments}

The authors are partially supported by the Spanish Ministry of Economy and Competitiveness, D.G.I. Project no. MTM2011-23843, and Junta de Andalucía Grant FQM3737.

\section{References}

[1] Y. Benyamini, S. Lassalle, and J. G. Llavona, "Homogeneous orthogonally additive polynomials on Banach Lattices," Bulletin of the London Mathematical Society, vol. 38, no. 3, pp. 459-469, 2006.

[2] D. Pérez-García and I. Villanueva, "Orthogonally additive polynomials on spaces of continuous functions," Journal of Mathematical Analysis and Applications, vol. 306, no. 1, pp. 97105, 2005.

[3] C. Palazuelos, A. M. Peralta, and I. Villanueva, "Orthogonally additive polynomials on $C^{*}$-algebras," Quarterly Journal of Mathematics, vol. 59, no. 3, pp. 363-374, 2008.

[4] K. Sundaresan, "Geometry of spaces of homogeneous polynomials on Banach lattices," in Applied Geometry and Discrete Mathematics, Discrete Mathematics and Theoretical Computer Science, pp. 571-586, American Mathematical Society, Providence, RI, USA, 1991.

[5] D. Carando, S. Lassalle, and I. Zalduendo, "Orthogonally additive polynomials over $C(K)$ are measures-a short proof," Integral Equations and Operator Theory, vol. 56, no. 4, pp. 597602, 2006.

[6] M. Burgos, F. J. Fernández-Polo, J. J. Garcés, and A. M. Peralta, "Orthogonality preservers revisited," Asian-European Journal of Mathematics, vol. 2, no. 3, pp. 387-405, 2009.

[7] Q. Bu and G. Buskes, "Polynomials on Banach lattices and positive tensor products," Journal of Mathematical Analysis and Applications, vol. 388, no. 2, pp. 845-862, 2012.
[8] D. Carando, S. Lassalle, and I. Zalduendo, "Orthogonally additive holomorphic functions of bounded type over $C(K)$," Proceedings of the Edinburgh Mathematical Society, vol. 53, no. 3, pp. 609-618, 2010.

[9] J. Á. Jaramillo, Á. Prieto, and I. Zalduendo, "Orthogonally additive holomorphic functions on open subsets of $C(K)$," Revista Matematica Complutense, vol. 25, no. 1, pp. 31-41, 2012.

[10] A. M. Peralta and D. Puglisi, "Orthogonally additive holomorphic functions on $\mathrm{C}^{*}$-algebras," Operators and Matrices, vol. 6, no. 3, pp. 621-629, 2012.

[11] Q. Bu, M. H. Hsu, and N. Ch. Wong, "Zero products and norm preserving orthogonally additive homogeneous polynomials on $\mathrm{C}^{*}$-algebras," Preprint.

[12] M. Burgos, F. J. Fernández-Polo, J. J. Garcés, J. M. Moreno, and A. M. Peralta, "Orthogonality preservers in $\mathrm{C}^{*}$-algebras, JB* algebras and $\mathrm{JB}^{*}$-triples," Journal of Mathematical Analysis and Applications, vol. 348, no. 1, pp. 220-233, 2008.

[13] A. C. Zaanen, "Examples of orthomorphisms," Journal of Approximation Theory, vol. 13, no. 2, pp. 192-204, 1975.

[14] B. E. Johnson, "Local derivations on $C^{*}$-algebras are derivations," Transactions of the American Mathematical Society, vol. 353, no. 1, pp. 313-325, 2001.

[15] S. Dineen, Complex Analysis on Infinite Dimensional Spaces, Springer, 1999.

[16] T. W. Gamelin, "Analytic functions on Banach spaces," in Complex Potential Theory, vol. 439 of NATO Advanced Science Institutes Series C, pp. 187-233, Kluwer Academic, Dodrecht, The Netherlands, 1994.

[17] M. Wolff, "Disjointness preserving operators on $C^{*}$-algebras," Archiv der Mathematik, vol. 62, no. 3, pp. 248-253, 1994.

[18] S. Sakai, $C^{*}$-Algebras and $W^{*}$-Algebras, Springer, Berlin, Germany, 1971.

[19] S. Goldstein, "Stationarity of operator algebras," Journal of Functional Analysis, vol. 118, no. 2, pp. 275-308, 1993.

[20] J. D. M. Wright, "Jordan C*-algebras," Michigan Mathematical Journal, vol. 24, pp. 291-302, 1977.

[21] D. Topping, "Jordan algebras of self-adjoint operators," Memoirs of the American Mathematical Society, vol. 53, 1965. 


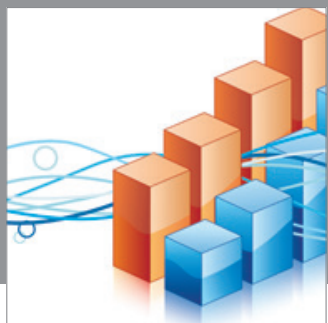

Advances in

Operations Research

mansans

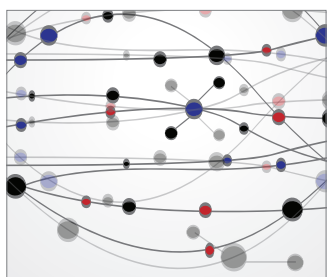

The Scientific World Journal
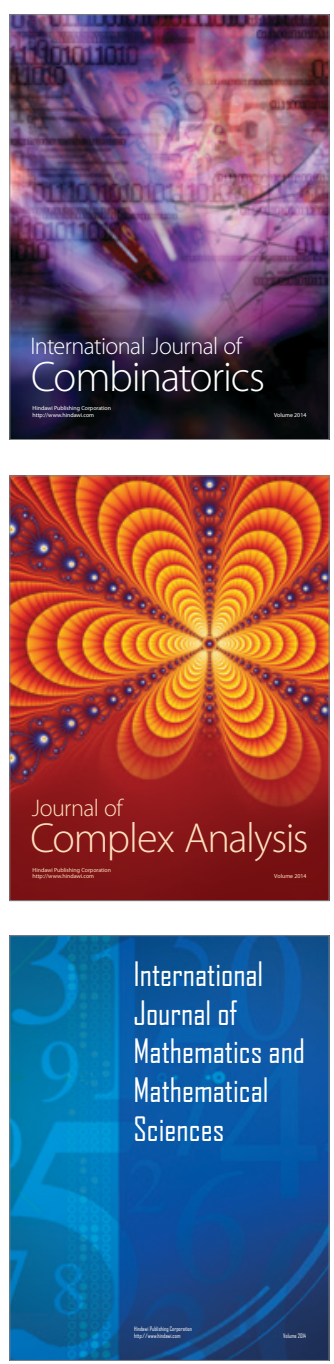
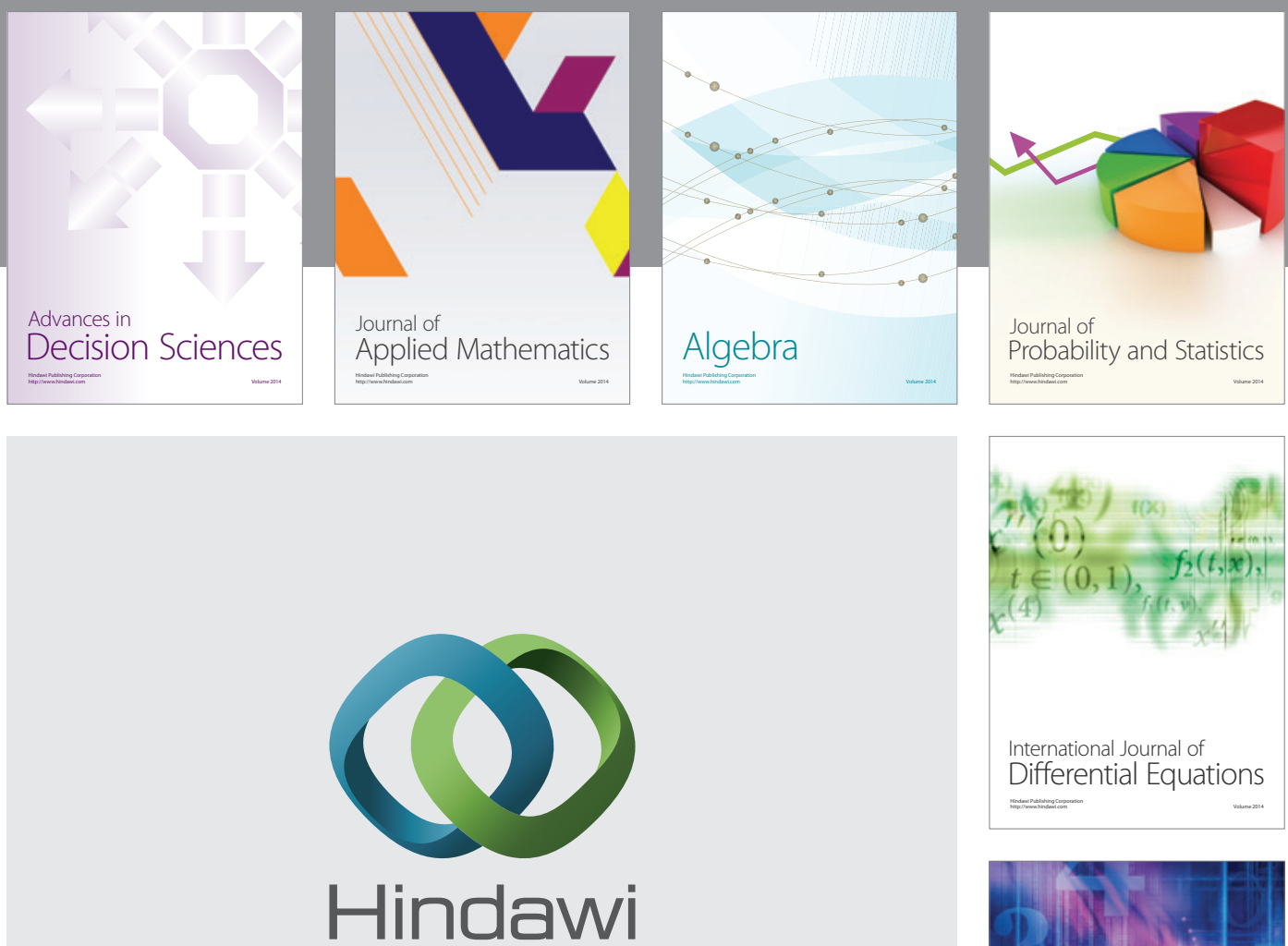

Submit your manuscripts at http://www.hindawi.com
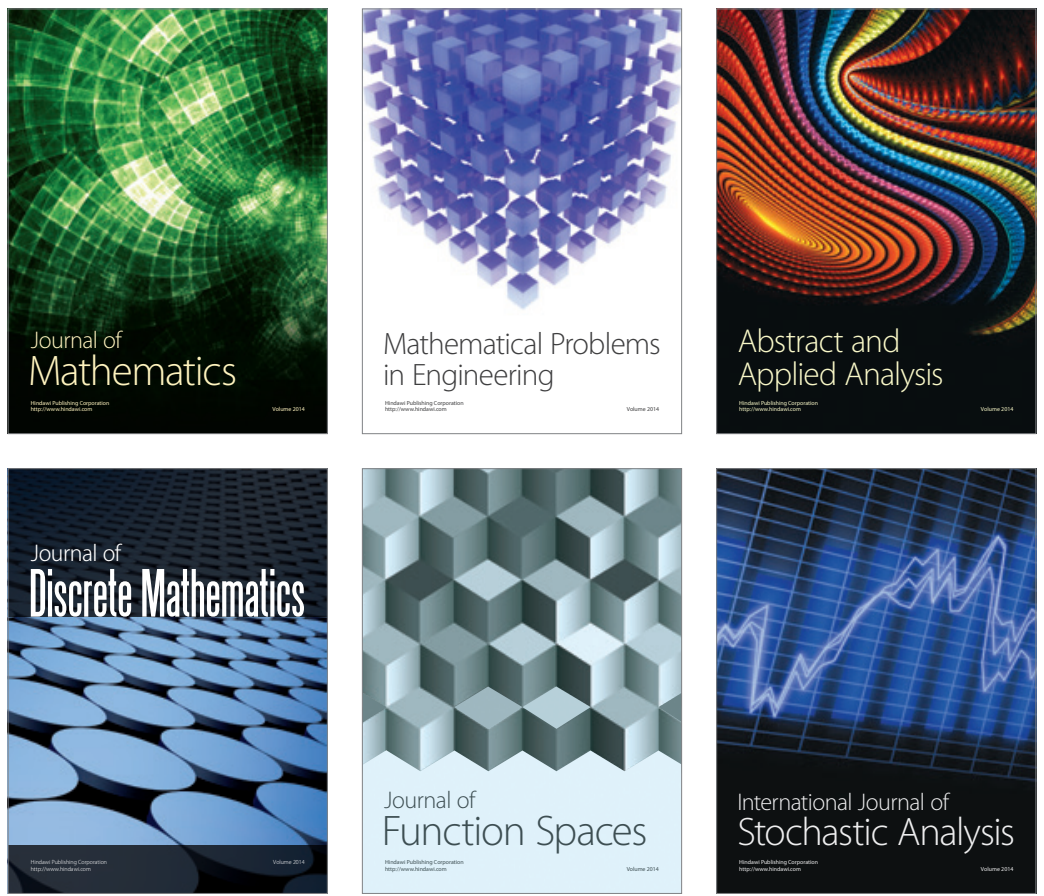

Journal of

Function Spaces

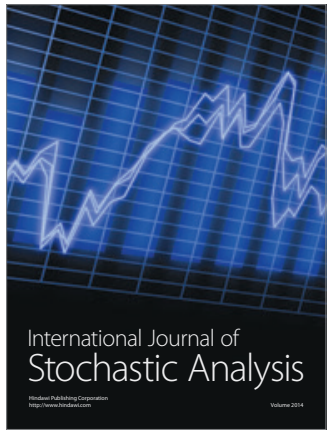

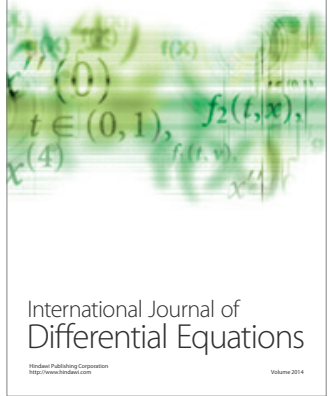
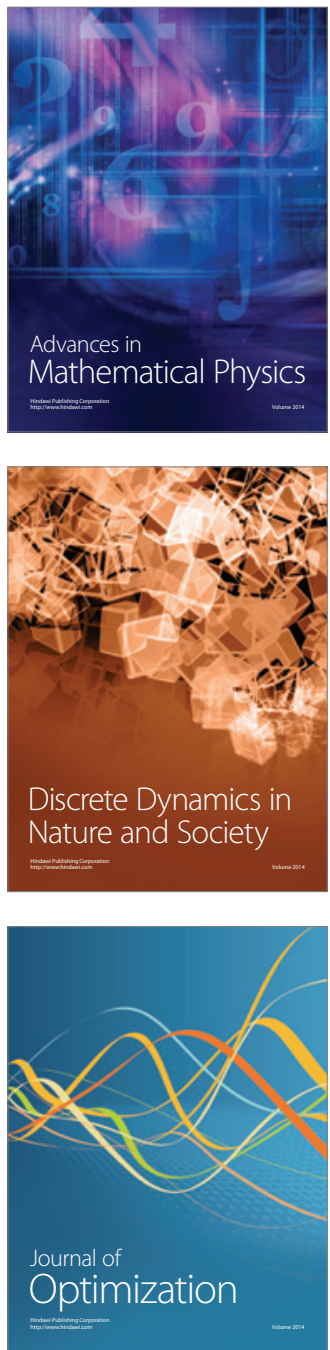